\title{
Aportes de la teoría feminista para (re)pensar el Estado
}

\section{Contributions of feminist theory to (re) think about the State}

ARTÍCULO

Recibido: junio de 2018

Aceptado: agosto de 2018

\section{Romina G. Amaya Guerrero}

Universidad Nacional de Quilmes. Departamento de Economía y Administración, Argentina.

Contacto: romina.amaya.guerrero@unq.edu.ar

\section{Resumen}

El presente trabajo consiste en aportar algunas claves de los debates feministas que contribuyan a enriquecer las discusiones sobre el Estado, especialmente visibilizando, en un plano teórico, el carácter patriarcal del Estado capitalista. La articulación propuesta tiene eje en los aportes del feminismo en diálogo con el marxismo, de las décadas del sesenta y setenta, particularmente, los trabajos enmarcados en lo que se denominó el debate sobre el trabajo doméstico. Conjugados con las discusiones provenientes de la revisión crítica de la teoría alemana de la derivación del Estado, es posible visibilizar teóricamente el carácter patriarcal del Estado, anclado en el trabajo doméstico y las relaciones de género allí puestas en juego.

Con el objetivo de descender el nivel de abstracción a este planteo, se analizan las relaciones de género manifestadas en las relaciones externas del Estado con el mercado, observando la evolución del trabajo doméstico, bajo la forma neoconservadora que asumió el Estado argentino durante la década del noventa y la metamorfosis posterior.

Este análisis permite observar un proceso de mercantilización de las relaciones sociales, a partir de la inserción laboral femenina, acompañado a su vez de un proceso de desestatalización, en el que el Estado dejó de hacerse cargo de determinados gastos sociales. Esto generó un reforzamiento de las relaciones de género, ya que las mujeres continuaron haciéndose cargo del trabajo doméstico.

Palabras claves: relaciones de género; trabajo doméstico; estado.

\begin{abstract}
The present work consists in providing some keys of the feminist debates contributing to enrich the discussions on the State, especially making visible, on a theoretical level, the patriarchal character of the capitalist State. The articulation proposed has an axis in the contributions of feminism in dialogue with Marxism, from the sixties and seventies, particularly, the works framed in what was called the debate on domestic work. Conjugated with the discussions stemming from the critical review of the German theory of the derivation of the State, it is possible to make visible theoretically the patriarchal character of the State, anchored in domestic work and the gender relations put into play there.

With the aim of lowering the level of abstraction to this proposal, the gender relations manifested in the external relations of the State with the market are analyzed observing the
\end{abstract}


evolution of domestic work, under the neoconservative form assumed by the Argentine State during the decade of the ninety and the subsequent metamorphosis.

This analysis allows us to observe a process of commodification of social relations, based on the female labor insertion, accompanied in turn by a process of de-nationalization, in which the State stopped taking charge of certain social expenditures. This generated a reinforcement of gender relations, since women continued to take charge of domestic work.

Keywords: gender relationships; domestic work; State

\section{Introducción}

Encaro en este trabajo un desafío, teórico y político, que consiste en conjugar las argumentaciones provenientes del feminismo marxista y de la revisión crítica de la teoría alemana de la derivación del Estado, que permiten poner de manifiesto, en un plano teórico, el carácter patriarcal del Estado capitalista.

La articulación propuesta tiene eje en los aportes del feminismo en diálogo con el marxismo en las décadas del 60 y 70 , especialmente los trabajos enmarcados en lo que se denominó el debate sobre el trabajo doméstico. Tempranamente, este debate "argumentó la necesidad de visibilizar el rol del trabajo doméstico no remunerado en el proceso de acumulación capitalista, y las implicancias en términos de explotación de las mujeres, tanto por parte de los capitalistas como de 'los maridos”' (Rodríguez Enríquez, 2015, p. 35).

El enfoque propuesto, a su vez, se complementa con los aportes provenientes de los análisis críticos de la teoría de la derivación. Así, considerando el Estado "no como una cosa en sí, sino como una forma social, una forma de las relaciones sociales" (Holloway, 1996 , p. 355) es válido preguntarse sobre la posibilidad de que las relaciones sociales entre varones y mujeres -0 , dicho de otra manera, las relaciones de género- se manifiesten en el Estado. Es decir, si existe tal cosa como un Estado patriarcal. Asimismo, la particularización en una forma de Estado que da cuenta de "las características que reviste el Estado capitalista en cierto período histórico y en uno o varios territorios nacionales particulares" (Bonnet y Piva, 2013, p. 3) abre el interrogante sobre las especificidades que adquieren esas relaciones de género en determinadas formas de Estado.

El recorrido que realizaré para llevar este desafío adelante inicia con la justificación de las elecciones teóricas en cuanto a la teoría feminista, especialmente alrededor del debate sobre el trabajo doméstico y las principales contribuciones provenientes de este debate. Luego, presentaré las posibilidades de articulación con la teoría del Estado y, en particular, observaré las manifestaciones de las relaciones de género a partir del vínculo del Estado y el mercado bajo la forma de Estado neoconservadora de Argentina de la década del 90 y la fase de metamorfosis del Estado iniciada a partir de la insurrección del 2001 (Bonnet y Piva, 2013). En el último apartado se presentan las reflexiones finales del trabajo. 


\section{Aportes de la teoría feminista: el debate sobre el trabajo doméstico}

En un diálogo crítico con la teoría marxista puede plantearse que la corriente feminista proveniente de esa adscripción teórica desarrolló numerosos aportes en el estudio de la inserción de las mujeres en la economía.

Los instrumentos del marxismo y el feminismo, adecuadamente entrelazados o desposados, como se decía entonces, bien podían dar pie a una crítica radical desde la economía política que pusiera en cuestión la contribución oculta de las mujeres en la casa y su importancia en el proceso de acumulación del capital" (Vega y Gutiérrez Rodríguez, 2014, p. 10).

Esta "crítica radical" aparece condensada en el debate sobre el trabajo doméstico, desarrollado en las décadas del 60 y 70, en un contexto de renovación del marxismo y de auge de los movimientos feministas (Carrasco, 1991). Con este debate, se rompe una tradición en la corriente marxista donde los temas estudiados sobre el trabajo femenino sólo se orientaban al trabajo asalariado. Las discusiones sobre el trabajo doméstico inauguran un nuevo centro de interés analítico que pronto convocó a distintas corrientes del feminismo y el marxismo. Si bien existen diversos aportes y perspectivas, la idea subyacente es estudiar el trabajo doméstico como contribución para entender la subordinación de la mujer en la sociedad capitalista. ${ }^{1}$ En este sentido, uno de los elementos esenciales que se mantiene en los trabajos es la relación entre trabajo doméstico no remunerado directamente y la reproducción social (Carrasco, 1991). A su vez, esta relación es la bisagra que permite la articulación de los aportes de este debate con aquellos sobre el Estado, tal como presentaré más adelante. Por este motivo, a continuación, profundizaré sobre el intercambio alrededor de ese tema.

De la elección teórica sobre el Estado, el aspecto principal que recorreré del debate sobre el trabajo doméstico es la relación que existe entre la esfera de producción doméstica y la esfera de la acumulación del capital. Mariarosa Dalla Costa en Las mujeres y la subversión de la comunidad (1977), editado por primera vez en 1971, es una de las primeras autoras que aborda directamente la cuestión de la reproducción de la fuerza de trabajo, entendiendo que ésta es la función específica de la mujer bajo el capitalismo (Carrasco, 1991).

Dalla Costa (1977) sostiene que la figura del "ama de casa" es la figura central del papel femenino en la división capitalista del trabajo y que todas las mujeres, incluso las que trabajan fuera de la casa, continúan siendo amas de casa. Cuando la autora se refiera a "todas las mujeres" hace referencia a todas las mujeres, sin importar las clases sociales, aunque diferencia las distintas formas en que se lleva adelante el trabajo doméstico; sin embargo, afirma que se concentrará en el papel del ama de casa de la clase obrera, 
entendiéndolo como determinante para la posición de las demás mujeres, "pero esto no implica que ella sea la única explotada" (Dalla Costa, 1977, p. 22).

La autora sostiene que, si bien la opresión de las mujeres es anterior al capitalismo, bajo este modo de producción la explotación fue más intensa. Así, recorre en las páginas de su artículo la conformación de la sociedad capitalista y hace foco en el rol de la mujer. Así, Dalla Costa (1977) sostiene que

el capital, al destruir la familia, la comunidad y la producción como un todo, ha concentrado, por un lado, la producción social básica en la fábrica y la oficina, y, por otro, ha separado al hombre de la familia y lo ha convertido en un trabajador asalariado" (p. 25).

Luego de la expulsión de los hombres, se produjo la expulsión de los niños hacia la escuela, y se despojó a la familia de su rol educativo. Así, quienes quedaron en el hogar fueron las mujeres.

En este contexto, Dalla Costa afirma que el fundamento de la sociedad capitalista es el trabajador asalariado y, sea hombre o mujer, se lo explota directamente. Así, podemos decir que según esta autora el salario es la forma fetichizada que revisten las relaciones sociales de explotación de clase. Sostiene, entonces, que el trabajo de las mujeres amas de casa (todas las mujeres), en apariencia por fuera de la relación salarial, pareciera un servicio personal fuera del capital, dejando a la mujer "atrapada dentro de las condiciones precapitalistas de trabajo" (Dalla Costa, 1977, p. 33), sin salario y atrapada en su hogar, en condiciones de aislamiento que moldean el mito de la incapacidad femenina. ${ }^{2}$ Aquí se presenta uno de los ejes de discusión dentro del debate, en relación con el salario para el trabajo doméstico. ${ }^{3}$

Silvia Federici es una pionera sobre el salario para el trabajo doméstico. En 1975 argumentó que "la condición no remunerada del trabajo doméstico ha sido el arma más poderosa en el fortalecimiento de la extendida asunción de que el trabajo doméstico no es un trabajo" (Federici, 2013, p. 37). En este sentido, plantea "no deberíamos desestimar la capacidad del salario para desmitificar nuestra feminidad y hacer visible nuestro trabajo" (Federici, 2013, p. 40).

Esta línea argumental -vinculada especialmente con la acción política del movimiento de mujeres- fue principalmente discutida desde las corrientes alineadas más estrechamente al feminismo donde se sostenía que percibir un salario por las tareas domésticas afianzaba el rol de la mujer en el ámbito del hogar.

Más allá de cuál fuera considerada la mejor táctica política para visibilizar el trabajo doméstico, lo que va dejando en claro este debate es la relevancia de estas tareas en el modo de producción capitalista. De acuerdo a Dalla Costa, las mujeres producen en una "fábrica social" y, ¿qué producen allí? Producen servicios sociales que sirven a la reproducción de las fuerzas de trabajo. La autora discute el posicionamiento general del 
marxismo que ubica a las mujeres como productoras de valores de uso consumidos al interior del hogar y afirma que el trabajo doméstico es una función esencial en la producción de plusvalía, lo que la lleva a considerar el trabajo doméstico como productivo -en el sentido marxista del término. Alrededor de esta afirmación, se establece una segunda discusión dentro del debate: el trabajo doméstico, ¿es o no trabajo productivo?

Wally Seccombe (2005 [1974]) afirma que el trabajo doméstico si bien es necesario, desde el punto de vista del marxismo, es improductivo. A continuación, presentaré la línea argumental de su artículo que le permite arribar a dicha conclusión.

En su trabajo Seccombe presenta un análisis detallado de la forma en que se inserta el trabajo doméstico en el modo capitalista de producción, reconociendo -al igual que Dalla Costa- que el tránsito del feudalismo al capitalismo implicó la escisión del trabajo (y de la fuerza de trabajo) en dos unidades: una unidad doméstica -de la que participan las mujeres- y una unidad industrial -de la que participan los hombres-. La función principal de la primera es reproducir la fuerza de trabajo, a diferencia de la segunda que produce mercancías (Carrasco, 1991). Este hecho implica, a su vez, que "el trabajador doméstico" (usando la formulación de Seccombe) se encuentra separado no sólo de los medios de producción -como es el caso del trabajador de la esfera industrial- sino de los medios de intercambio, lo que genera dependencia del acuerdo privado que establece con su "marido" en cuanto a la redistribución del salario. En este contexto, el autor está en condiciones de afirmar que la esfera doméstica está por fuera de la relación salarial presente en la esfera industrial- por lo que el "trabajo doméstico es un asunto de carácter privado y de su exclusión de la escena de la apropiación del excedente es que la ley del valor no rige sobre el trabajo doméstico" (Seccombe, 2005, p. 180). La última característica de la relación entre la familia y la producción que señala Seccombe, tiene que ver con la separación de la esfera de la producción de la del consumo, a la que se interpuso el mercado de mercancías.

A partir de esta caracterización, Seccombe analiza la relación entre el trabajo doméstico (específicamente el ama de casa en tanto trabajadora) y el capital. El autor sostiene que la fuerza de trabajo opera como mediadora en esta relación, ya que se halla enlazada hacia atrás con el trabajo doméstico y hacia adelante con el capital (en su forma equivalente, el salario). A partir del análisis de la función del trabajo doméstico, que consiste en transformar los productos para que adquieran una forma consumible, Seccombe (2005) concluye que

cuando el ama de casa actúa directamente sobre los bienes adquiridos con el salario y altera necesariamente la forma de éstos, el trabajo de ella se transforma en parte de la masa congelada de trabajo pasado corporizado en la fuerza de trabajo. El valor creado por el ama de casa es realizado como una parte del valor que la fuerza de trabajo adquiere como mercancía cuando dicha fuerza de trabajo es vendida" ( $p$. 184). 
Así, el salario en apariencia paga por el trabajo realizado en el sitio donde se lleva a cabo, pero, en realidad, paga por el trabajo reproductor de la fuerza de trabajo de toda la familia, afirmación que genera nuevas discusiones en el debate, pero que no serán abordadas en el presente trabajo.

De acuerdo con Seccombe, entonces, el trabajo doméstico transfiere y crea valor. Sin embargo, el autor sostiene que estas características no implican que pueda ser considerado productivo en términos marxistas. Afirma, citando a Marx, que el trabajo productivo es aquél que "es realizado en relación directa con el capital y produce valor excedente y el trabajo doméstico no satisface ninguno de estos dos criterios" (Seccombe, 2005 , p. 188) ya que se intercambia con el salario que percibe "el marido", que constituye el ingreso del hogar. Y no produce valor excedente, ya que el valor del trabajo doméstico se transfiere a la fuerza de trabajo y termina formando parte de su valor.

Las respuestas a este enunciado de Seccombe no se hicieron esperar. Jean Gardiner (2005) publica en 1975 un artículo con dos objetivos específicos: discutir el argumento de Seccombe y examinar dos problemas referentes al trabajo doméstico de las mujeres referentes a por qué el trabajo doméstico y el cuidado de los niños es responsabilidad de las mujeres y se mantiene organizado en forma privada así como cuáles son las presiones que actúan en favor y en contra de una transformación en la familia.

Sobre la crítica que realiza Gardiner, me interesa resaltar dos puntos. En primer lugar, la autora sostiene que en el trabajo de Seccombe no aparecen indicios de que el capital esté interesado en que las mujeres realicen, o no, el trabajo doméstico. Es decir, de acuerdo con la explicación de este autor, en términos de la generación de plusvalía, el capital no gana ni pierde con el trabajo doméstico, por lo tanto, no habría incentivos para querer conservarlo. Sin embargo, desde el punto de vista de Gardiner, el capital sí tiene interés, para garantizar la acumulación capitalista, en el tiempo que destinan las mujeres al trabajo doméstico (amas de casa de tiempo completo, medio tiempo o cuánto tiempo son asalariadas).

Luego, y derivado de la crítica anterior, Gardiner sostiene que Seccombe no muestra cómo puede volverse significativo el trabajo doméstico en el capitalismo en un momento de crisis, como el que caracteriza el tiempo en que ella escribe. Utiliza un ejemplo para reforzar su argumento: si se reduce el salario del trabajador, en términos de Seccombe significaría, entonces, que se reduce el valor creado por el trabajo doméstico. De acuerdo a Gardiner, esta parecería una conclusión equivocada.

La autora pasa, entonces, a enfocar su análisis en las causas que han conservado el trabajo doméstico, observando que en el capitalismo este tipo de trabajo tiene dos aspectos importantes. En primer lugar, un requisito histórico del modo capitalista de 
producción fue que la economía doméstica familiar de los trabajadores dejase de ser autosuficiente y autorreproductora. El segundo aspecto del trabajo doméstico que realizan las mujeres es que en todas las etapas del desarrollo del capitalismo ha desempeñado un papel fundamental, aunque cambiante, en la satisfacción de las necesidades de los trabajadores. Así, "se ha conservado el trabajo doméstico para realizar una parte importante de la reproducción y la conservación de la fuerza de trabajo" (Gardiner, 2005 p. 99). La autora identifica tres factores posibles para que esto sea así: económicos, psicológicos e ideológicos.

Me referiré a los aspectos económicos señalados por Gardiner. Así, a diferencia de Seccombe, Gardiner sostiene que, en Marx, el valor de la reproducción de la fuerza de trabajo no incluye el trabajo doméstico y está restringido a las mercancías compradas por el salario y consumidas por la familia del trabajador. A su vez, Gardiner sostiene que el trabajo doméstico contribuye a la extracción de plusvalor, ya que mantiene el trabajo necesario a un nivel más bajo que el nivel efectivo de subsistencia de la clase trabajadora. Así,

el trabajo doméstico no crea valor según la definición de valor adoptada por Marx, sin embargo, sí contribuye al plusvalor al mantener el trabajo necesario, o el valor de la fuerza de trabajo, a un nivel más bajo que el nivel efectivo de subsistencia de la clase trabajadora (Gardiner, 2005, p. 107).

Es decir,

al no incorporar este trabajo doméstico en el valor de la fuerza de trabajo, se la mantiene en un valor menor del que realmente le correspondería. Esta diferencia hace que la plusvalía se vea aumentada, aunque esto no significa que se extraiga plusvalía del trabajo doméstico (Carrasco, 1991, p. 215).

Esta tesis pasó a ser la más generalizada y aceptada por los autores y autoras que, posteriormente, se involucraron en el debate sobre el trabajo doméstico.

Siguiendo, entonces, esta formulación,

la contribución que el trabajo doméstico realiza a la producción de plusvalía es la de mantener el valor de la fuerza de trabajo por debajo del costo de su reproducción. El mecanismo para que esto ocurra consiste en la retención dentro del hogar de aquellos aspectos de la reproducción y el mantenimiento de la fuerza de trabajo que no son rentables ni para la producción capitalista, ni para el Estado, en caso de que éste eventualmente se hiciera cargo. La configuración futura del trabajo doméstico dependería entonces de la interrelación de los costos de la reproducción de la fuerza de trabajo, el proceso de acumulación capitalista y la demanda de trabajo femenino remunerado (Marco Navarro y Rodríguez Enríquez, 2010, p. 100). 
Así, esta relación entre trabajo doméstico y acumulación capitalista introduce los lineamientos generales que permitirán articular los aportes generados en este debate a la teoría del Estado, y reflexionar sobre las posibles implicancias de esta intersección.

\section{El desafío: la articulación con el Estado}

Al inicio del trabajo me propuse el desafío de plantear las posibilidades que brindan los aportes del feminismo en diálogo con el marxismo para incorporar al Estado la dimensión de las relaciones de género. Me referí a los aportes del debate sobre el trabajo doméstico que desarrollan ampliamente la relación del trabajo doméstico con la acumulación capitalista, y su rol clave en garantizarla. Sin embargo, no se encuentra un planteo claro sobre la dimensión genérica del trabajo doméstico, ${ }^{4}$ a pesar de que en todos ellos se da por sentado que quienes lo realizan son mujeres. Incorporar el género como categoría específica resulta fundamental para arribar a la articulación teórica que permite develar el carácter patriarcal del Estado. Es necesario, pues, incorporar al análisis las relaciones de género. Sigo aquí la definición de género de Scott (1996), quien considera que

el género es un elemento constitutivo de las relaciones sociales basadas en las diferencias que distinguen los sexos y el género es una forma primaria de relaciones significantes de poder. (...) Como elemento constitutivo de las relaciones sociales basadas en las diferencias percibidas entre los sexos, el género comprende cuatro elementos interrelacionados: primero, símbolos culturalmente disponibles que evocan representaciones. (...) Segundo, conceptos normativos que manifiestan las interpretaciones de los significados de los símbolos (...) (p. 277).

El tercer elemento lo constituyen las instituciones sociales, como el parentesco, la familia, pero también instituciones como el mercado de trabajo, la educación y la política. $Y$ el cuarto aspecto del género es la identidad subjetiva (Scott, 1996).

De esta manera, la relación entre trabajo doméstico y acumulación capitalista está enmarcada en las dimensiones que señala Scott en las que opera el género, y que se manifestarán en el carácter de Estado. El trabajo doméstico constituye la bisagra que permite articular ambas teorías y condensa las relaciones de género que aseguran la acumulación capitalista. Por este motivo, siguiendo la revisión crítica de la teoría de la derivación, estas relaciones se manifestarán en el Estado. Antes de desarrollar los aportes teóricos que permiten esta articulación, me parece conveniente destacar los trabajos de las corrientes feministas que, desde distintas perspectivas, abordan la cuestión del Estado.

Pateman (1988) y MacKinnon (1989), provenientes de distintas corrientes del feminismo, la primera vinculada al feminismo socialista y la segunda al feminismo radical, ofrecen un enfoque instrumentalista del Estado, como se evidencia en esta cita de Valobra (2015) refiriéndose a los mismos: 
el Estado no sólo se define por los intereses que sirve o por quiénes detentan el poder sino porque está garantizando ciertas relaciones sociales que, además de vehiculizar la existencia de ciertos modos de producción capitalistas, también supone relaciones generizadas de subordinación que impiden impugnar el sistema (p. 39).

Más recientemente, los aportes de Nancy Fraser (2015) resultan sustanciales en cuanto a la articulación y las estrategias del feminismo como movimiento social y de lucha, y la necesidad de una conjugación de reivindicaciones vinculadas a la distribución y al reconocimiento. Analiza, especialmente, cómo el feminismo organiza estas demandas en dos contextos distintos del capitalismo: durante el capitalismo organizado desde el Estado (en los Estados de bienestar, que Fraser denomina Estado keynesiano-westfaliano) y, posteriormente, durante el neoliberalismo. Este segundo contexto implica el retroceso tanto en las funciones de bienestar como en el asentamiento del territorio nacional (aspecto ligado a lo "westafliano") ante un avance globalizador.

Si bien Fraser (2015) describe con precisión las transformaciones que implican estos dos contextos, a nivel de la acumulación de capital y de la lucha feminista, el Estado aparece como "una cosa", como una cuestión externa y aparece definido a partir de sus funciones. Específicamente, Fraser refiere a los aspectos vinculados a la justicia y a las reivindicaciones en torno a ella, y desarrolla un concepto de justicia tridimensional ligado a la redistribución, el reconocimiento y la representación. La primera refiere a una dimensión económica, la segunda al estatus y la tercera a la política.

Los aportes de Fraser son fundamentales para la discusión de las demandas del feminismo, y bien podrían complementarse con una teoría del Estado -como la que abordaremos a continuación- que no lo ubique como una cuestión externa, "como una cosa en sí, sino como una forma social, una forma de las relaciones sociales" (Holloway, 1995 , p. 355), tal como propone la revisión crítica de la teoría de la derivación. En este marco, es posible pensar las relaciones de género dentro de esta dimensión de las relaciones sociales. Siguiendo a Hirsch (2005):

El surgimiento de la sociedad capitalista, del trabajo asalariado, de la libertad ciudadana, la democracia y el Estado han originado un "patriarcado capitalista", en el cual se modificaron no sólo los contenidos y mecanismos de la opresión, sino también las condiciones de la liberación [de las mujeres]. Precisamente la división capitalista entre 'Estado' y 'sociedad' ha generado la contraposición entre 'lo público' y 'lo privado', que hoy está considerada con razón como uno de los mecanismos decisivos para la opresión de género (...) Esto significa por cierto, que la relación entre 'sociedad' y 'Estado' no solo es determinada por el proceso capitalista de revaloración, sino que también estrechamente ligada a él, por las relaciones sexuales, étnicas, nacionalistas y racistas de opresión y explotación. El Estado burgués es entonces siempre y simultáneamente un Estado capitalista, racista y patriarcal y las relaciones sociales que él expresa y 'regula', mediante su aparato, comprenden todas estas contradicciones (Hirsch, 2005, p. 171). 
El trabajo doméstico en tanto trabajo no remunerado del cual el capital se beneficia incrementando sus niveles de plusvalía al pagar la fuerza de trabajo por debajo de su valor de reproducción, opera tanto en el proceso de revaloración del capital, como en la dimensión de las relaciones sexuales, en la determinación de la relación de la sociedad y el Estado. Desde el punto de vista de los aportes vinculados al debate británico de la reformulación del Estado (debate del cual Hirsch forma parte) esta determinación no sería mecánica. Así,

las relaciones entre Estado y capital no deben entenderse en términos de la determinación más o menos mecánica de una superestructura por parte de una estructura económica ni tampoco de la autonomía relativa de esa superestructura, argumentó Holloway en este sentido, sino en términos de sendas formas diferenciadas y fetichizadas de unas mismas relaciones sociales capitalistas signadas por el antagonismo de clase (Bonnet, 2007, p. 287).

Ahora bien, llegado este punto me parece propicio introducir el concepto de forma de Estado, también proveniente de esta revisión crítica de los debates de la derivación. Esta noción

rinde cuenta de las características que reviste el Estado capitalista en cierto período histórico y en uno o varios territorios nacionales particulares, características asociadas a su vez con un conjunto de instituciones que constituyen los aparatos de Estado y con un conjunto de actividades que constituyen las funciones de estos estados. Las distintas formas de estado se estructuran a partir de los distintos modos en que se articula la unidad-en-la-separación entre lo político y lo económico que es constitutiva del Estado capitalista. Estas relaciones entre lo político y lo económico determinan las formas de Estado en dos sentidos: como relaciones externas al Estado (es decir, como relaciones entre el Estado y el mercado) y como relaciones interiorizadas en el propio Estado (como relaciones entre los distintos poderes del estado, en un sentido amplio) (Bonnet y Piva, 2013, p. 3).

La articulación propuesta sugiere que las relaciones de género, condensadas en el trabajo doméstico no remunerado que realizan las mujeres, se manifiestan en la forma Estado, configurando el Estado capitalista como un Estado patriarcal, como plantea Hirsch (2005). De esta manera, se espera que en una forma de Estado determinada, correspondiente a una modalidad específica de acumulación capitalista (Bonnet, 2007 en referencia a Hirsch), se manifiesten estas relaciones de género en los aparatos de Estado, las funciones de Estado, en las relaciones externas al Estado con el mercado e internas entre sus poderes.

Para aclarar esta afirmación es conveniente analizar una forma de Estado específica. En particular, me referiré a la forma de Estado neoconservadora o neoliberal que asumió el Estado argentino en los 90 y la metamorfosis que experimentó esta forma de Estado a partir de la crisis y la insurrección de diciembre de 2001 (Bonnet y Piva, 2013). Observaré la dimensión de género a partir de las relaciones externas al Estado, es decir entre el 
Estado y el mercado, y me enfocaré en dos aspectos vinculados al trabajo doméstico, pieza fundamental de la acumulación capitalista. Por un lado, la inserción de las mujeres en el mercado de trabajo y su correlación con el tiempo "exclusivo" de las mujeres como amas de casa. Por el otro, las características generales de la economía del cuidado ${ }^{5}$ de niños y niñas, donde se ponen de manifiesto la estatalización y la mercantilización de esta forma de Estado específica.

Según Bonnet (2011), las relaciones entre Estado y mercado no serán tomadas como antagónicas, como han sugerido diversas teorías, sino que "el Estado y el mercado capitalistas son formas conceptual e históricamente diferenciadas de unas mismas relaciones sociales. El antagonismo entre capital y trabajo es inherente a estas relaciones sociales" (p. 19). Así, la estatalización, como proceso de ampliación de las relaciones estatales y la mercantilización, como proceso de ampliación de las relaciones mercantiles, son procesos que no necesariamente son inversos.

El modo de acumulación neoconservador, que implicó que el Estado asumiera una forma neoconservadora, supuso una mayor integración en el mercado mundial, lo que

impuso la presión constante hacia el incremento de la explotación del trabajo, ya sea a través del aumento de la productividad (en condiciones expansivas) o del recorte del salario nominal (en condiciones recesivas), como requisito de la inserción exitosa del capitalismo argentino (...) El proceso de acumulación, en consecuencia, descansó cada vez más en la reducción de salarios nominales y en la extensión e intensificación de la jornada laboral como mecanismos para mantener niveles de competitividad y de rentabilidad del capital local compatibles con un proceso sostenido de inversión (Bonnet y Piva, 2009, p. 11 y 12).

En este contexto, se observa una mayor incorporación de las mujeres al mercado de trabajo, proceso entendido como una mercantilización de las relaciones sociales (Bonnet, 2011). Así, mientras que en los inicios de la Convertibilidad el $36,76 \%$ de las mujeres mayores de 15 años participaba de la actividad económica, ${ }^{6}$ en el 2001 este porcentaje se había elevado a 45,16\% (CEPALSTAT). Este proceso continuó luego de la insurrección del 2001, y en el año 2015 el 53\% de las mujeres participaba de la actividad económica. Principalmente, este proceso se atribuye a las estrategias familiares adoptadas en el contexto descrito anteriormente, de caída de salarios y aumento del desempleo (MTEySS, $\mathrm{s} / \mathrm{f})$.

En este proceso, es de gran importancia observar las características de la inserción de las mujeres en la actividad económica. Así, Heller (2000) afirma que existe un proceso de segregación horizontal, que implica ocupaciones primarias reservadas para los varones y otras secundarias netamente femeninas, de bajo prestigio social, con inferiores remuneraciones y condiciones de trabajo más desfavorables. Esto se evidencia en las elevadas tasas de participación femenina en sectores como educación y salud (enfermeras, especialmente). A su vez, estos sectores están estrechamente ligados a los 
estereotipos de género que asocian a las mujeres con tareas de cuidado y mantienen salarios bajos en comparación con otros sectores de servicios.

Existe otro proceso que caracteriza la inserción laboral de las mujeres, que tiene que ver con la segregación vertical, que se expresa fundamentalmente por pautas diferenciadas de distribución dentro del mercado de trabajo según categoría ocupacional, y donde la escasa presencia femenina en los puestos ejecutivos y directivos es una constante (Heller, 2000). Se suele atribuir a este fenómeno el concepto de "techo de cristal".

La segregación horizontal y vertical caracterizaron al mercado de trabajo tanto en el período de la convertibilidad como en la metamorfosis posterior que sufrió la forma de Estado.

La incorporación de las mujeres al mercado de trabajo remunerado, sin embargo, no las desligó del trabajo doméstico: el $76 \%$ de estas tareas es realizada por mujeres (INDEC, 2013). Así, si bien se redujo el tiempo exclusivo de las mujeres como amas de casa, continuaron a cargo del trabajo doméstico, cuestión que está estrechamente vinculada al punto siguiente.

Como mencioné más arriba, la otra dimensión de la relación entre el Estado y el mercado vinculada al trabajo doméstico que voy a observar tiene que ver con las características generales de la economía del cuidado, especialmente en el aspecto que hace a la absorción por parte del Estado de servicios del cuidado.

Un rasgo característico de la forma de Estado neoconservadora de los noventa está asociada a la mercantilización con desestatalización de las relaciones sociales. Más arriba quedó reflejado el proceso de mercantilización en relación con la incorporación de las mujeres al mercado de trabajo. Por su parte, el proceso de desestatalización originó interpretaciones del Estado como un Estado mínimo, lo que no se sostiene si se observan algunas cuestiones como el "tamaño" del Estado (medido en cantidad de dependencias, personal o presupuesto) (Bonnet, 2011). Lo que sí sucedió en esta metamorfosis de la forma de Estado, es un proceso de "descentralización hacia los niveles provinciales y municipales de la educación, la salud y la asistencia social" (Bonnet y Piva, 2009, p. 13). Y aquí aparece la clave para delinear las características de la economía del cuidado.

En términos de la organización social del cuidado, esta descentralización se implementó a través de la Ley Federal de Educación, que implicó el traslado de los niveles educativos primario y secundario a las provincias (el nivel primario ya había iniciado su traspaso en la década del 70 y culminó en esta década, mientras que el nivel secundario se transfirió en esta etapa). Así, cada provincia pasó a tener potestad de sus propios regímenes educativos. En clave de cuidados, y pensando especialmente en los niños/as más pequeños/as, esta Ley incorporó la obligatoriedad del pre-escolar (5 años), lo que 
significó apertura de estas salas. Sin embargo, no fue hasta principios del año 2015 que se amplió la obligatoriedad a los 4 años.

En este contexto, los espacios de cuidado para los niños/as más pequeños/as son de gestión privada, es decir, están enmarcados en la mercantilización. Esta característica, a su vez, no fue modificada con los cambios introducidos en 2015 , es decir que se mantiene hasta hoy. Si bien algunas jurisdicciones cuentan con la provisión de este tipo de servicios por parte del Estado local, en líneas generales se mantiene desde el Estado una concepción que ubica a las mujeres en su rol tradicional de madres y cuidadoras.

Como señala Zibecchi (2014)

existen investigaciones que demuestran que la organización social del cuidado en su conformación actual, en América Latina en general y en la Argentina en particular, es injusta. Esta injusticia en la organización y distribución se basa en que las responsabilidades de cuidado se encuentran desigualmente distribuidas entre familias, Estado, mercado y organizaciones comunitarias, por un lado, y entre varones y mujeres, por el otro. De modo que gran parte de las responsabilidades son asumidas por las familias, y dentro de ellas, por las mujeres casi en soledad con una intervención del Estado en la provisión de políticas muy específicas (por ejemplo, a través de la educación escolar obligatoria) (p. 21).

Estas afirmaciones se sostienen, por ejemplo, con los resultados de las Encuestas de Uso del Tiempo, que muestran que, en promedio, las mujeres dedican a las actividades vinculadas al cuidado el doble de tiempo que los varones. A su vez, cuando las mujeres se insertan en el mercado laboral, continúan efectuando estas actividades de cuidado, renunciando a tiempos de descanso y ocio. En los hogares donde ambos cónyuges participan en el mercado laboral, la distribución de responsabilidades de cuidado sigue estando concentrada mayormente en el tiempo de las mujeres (Zibecchi, 2014).

Así, en esta dimensión de la relación entre el Estado y el mercado también se observa un refuerzo de los roles de género, donde las tareas domésticas están principalmente a cargo de las mujeres, en una desigual división de tareas dentro del hogar. La mercantilización de estas relaciones sociales implica la satisfacción de estas necesidades de cuidados en el seno del mercado (llevada a cabo también por mujeres en tanto cuidadoras remuneradas), aunque también implica una familiarización de estas tareas, asentada en mujeres.

A lo largo de este apartado pudimos conjugar los aportes del debate sobre el trabajo doméstico provenientes del feminismo marxista con los aportes de la revisión crítica de la teoría alemana de la derivación del Estado. La articulación es posible gracias a que coinciden en anclar sus análisis en las características de la sociedad capitalista y a observar los mecanismos de la acumulación de capital. Así, el trabajo doméstico es fundamental para la acumulación, manteniendo el valor de la fuerza de trabajo por debajo del que realmente correspondería, y manifiesta unas relaciones de género desiguales, 
donde las mujeres aparecen como las principales responsables de garantizarlo. A su vez, esas relaciones de género se manifiestan en el Estado, haciendo del Estado capitalista necesariamente un Estado patriarcal. En los aportes de la revisión crítica de la teoría de la derivación, el Estado asume formas particulares en distintos períodos históricos y se estructuran a partir de los distintos modos de articulación entre lo económico y lo político.

Se analizó, entonces, el carácter patriarcal del Estado en la forma de Estado neoconservadora de Argentina durante la década del noventa, a partir de observar el trabajo doméstico como dimensión clave de ese Estado patriarcal. Se eligió trabajar en la relación externa del Estado, es decir su relación con el mercado, para lo cual se hizo énfasis en dos dimensiones: la inserción de las mujeres en el mercado de trabajo, lo que implica menos mujeres como amas de casa de tiempo completo, y algunos rasgos de la economía del cuidado, refiriendo a un aspecto particular del trabajo doméstico vinculado al cuidado de niños/as.

¿Qué se pone de manifiesto cuando observamos el trabajo doméstico de las mujeres argentinas? Que, en la forma neoconservadora del Estado argentino y la metamorfosis seguida luego de 2001, las mujeres participaron en mayor porcentaje del mercado de trabajo, en ocupaciones específicas con menor remuneración y sin dejar de hacerse cargo de las tareas domésticas, lo que implica una mercantilización de las relaciones sociales y un doble provecho del capital. A su vez, el cuidado también continuó a cargo de mujeres, e incluso hubo un avance en la desestatalización de estas tareas.

Todo esto resulta en reforzar el carácter patriarcal del Estado. Sin embargo, esto no ha sido sin lucha y enfrentamiento por parte de los movimientos de mujeres y feministas que, en el período más reciente y a partir de los "paros de mujeres", pudieron visibilizar la desigual distribución de las tareas en el seno del hogar. Se abre aquí una interesante línea de análisis que queda pendiente para trabajos futuros.

\section{A modo de reflexión}

Al inicio de este trabajo me propuse incorporar aportes de la teoría feminista que pudieran ser articulados con una teoría del Estado cuyo énfasis no está en considerar el Estado como una "cosa" en sí misma o de definirlo a partir de sus funciones, sino como la fetichización de las relaciones sociales bajo el capitalismo. Relaciones sociales basadas en el antagonismo del capital y el trabajo.

En esa senda, seleccioné los aportes del debate sobre el trabajo doméstico, donde se expresa un fluido diálogo entre el feminismo y el marxismo, y se hizo foco en la relación existente entre el trabajo doméstico y la acumulación capitalista. Este debate permitió hacer visible aquello que era invisible hasta entonces, tanto en la práctica política como académica, el trabajo no remunerado de las mujeres en el seno del hogar. 
Tomando estos aportes fue posible articularlos con las teorías del Estado provenientes de la revisión crítica de los debates de la derivación alemana. Así, con el concepto de forma de Estado y a partir de los aportes realizados sobre el estudio de la forma de Estado neoconservadora de Argentina en la década de los 90 y la metamorfosis que sufrió después del 2001, se llevó a cabo el entrelazamiento de estas dos teorías. Especialmente desarrollé la relación entre Estado y mercado, y apunté dos temas: la incorporación de las mujeres en el mercado de trabajo y algunos rasgos generales sobre la economía del cuidado, especialmente en lo referido a niños/as.

Este ejercicio permitió corroborar los rasgos patriarcales que tiene la forma Estado capitalista (Hirsch, 2005), reforzados en la forma de Estado neoliberal y la metamorfosis posterior, a través de distintos mecanismos como la creciente participación de las mujeres en el mercado de trabajo, una economía del cuidado basada en la "familia", y tantas otras no volcadas en este trabajo.

Como dije al inicio, este desafío tuvo rasgos teóricos y políticos. Los teóricos se han señalado a lo largo de estas páginas. Los políticos han implicado la centralidad de la participación de las mujeres en la lucha de clases. Así, las motivaciones de las feministas en diálogo con el marxismo, buscando la incorporación de las mujeres en la lucha social y política de su tiempo, siguen siendo tan válidas hasta hoy.

\section{Notas}

${ }^{1}$ Es importante destacar que los autores y autoras que intervienen en el debate sobre el trabajo doméstico tienen, en general, una preocupación política por las formas y posibilidades de incorporación de las mujeres en la lucha social, política y revolucionaria. En este sentido, encontrar las causas de la posición de subordinación de las mujeres en la sociedad capitalista, tiene como objetivo principal desatar la rebeldía de las mujeres a esa subordinación y sumarlas en la lucha por la transformación de la sociedad.

${ }^{2}$ Dalla Costa (1977) habla de un "mito" porque sostiene que las distintas luchas sociales que ha llevado adelante la clase obrera, "la base ha sido siempre la organización informal ininterrumpida de las mujeres en la comunidad" y "en las luchas en el ciclo de producción directa, el apoyo y la organización de las mujeres, formal e informal, han sido decisivos" (Dalla Costa, 1977, p. 35).

${ }^{3}$ Dalla Costa es promotora de la Campaña Salario para el Trabajo Doméstico (Wages for Housework), desarrollada en Italia en 1972 junto a Silvia Federici, Leopoldina Fortunatti y Selma James.

${ }^{4}$ De acuerdo a algunas autoras, el debate sobre el trabajo doméstico no aportó especificidades sobre las relaciones entre varones y mujeres, sino que se ocupó de discutir sobre las relaciones del trabajo doméstico, principalmente realizado por mujeres, y la acumulación del capital: "En el "debate del trabajo doméstico" no se exploró el problema de las relaciones de género ni se preguntó si no serían los hombres, además de, o en lugar de los capitalistas, quienes se benefician del trabajo doméstico. Asimismo, se prestó poca atención al hecho de que es la combinación de trabajo doméstico y trabajo remunerado, más que el trabajo doméstico solo, lo que caracteriza a la experiencia de las mujeres en el capitalismo. En el "debate sobre el patriarcado" se discutieron algunos de estos elementos" (Rodríguez Enríquez, 2012 p. 27). La conjunción de estos debates permitió que la economía feminista articulara los aportes del primer debate con el segundo, realizando "importantes contribuciones al estudio del trabajo no remunerado, resaltando sus aspectos de género, su invisibilidad y su aporte central a la reproducción social y el funcionamiento de la economía" (Rodríguez Enríquez, 2012, p. 28).

${ }^{5}$ Tomo el concepto de "economía del cuidado" en un sentido amplio, considerando que "refiere a todas las actividades y prácticas necesarias para la supervivencia cotidiana de las personas 
en la sociedad en que viven. Incluye el autocuidado, el cuidado directo de otras personas (la actividad interpersonal de cuidado), la provisión de las precondiciones en que se realiza el cuidado (la limpieza de la casa, la compra y preparación de alimentos) y la gestión del cuidado (coordinación de horarios, traslados a centros educativos y a otras instituciones, supervisión del trabajo de cuidadoras remuneradas, entre otros). El cuidado permite atender las necesidades de las personas dependientes, por su edad o por sus condiciones/capacidades (niños y niñas, personas mayores, enfermas o con algunas discapacidades) y también de las que podrían autoproveerse dicho cuidado" (Rodríguez Enríquez, 2015, p. 36).

${ }^{6}$ Población económicamente activa (mujeres) de 15 años de edad y más como porcentaje del total de las mujeres de 15 años de edad y más (CEPALSTAT).

\section{Referencias bibliográficas}

Bonnet, A (2007). Estado y capital. Debates sobre la derivación y la reformulación del estado. En: M. Thwaites Rey (comp.). Estado y marxismo. Buenos Aires: Prometeo.

Bonnet, A. (2009). Las relaciones con el Estado en las luchas sociales recientes. Un planteo del problema a partir de la experiencia argentina. En: Bonnet, A. y A. Piva. Argentina en pedazos. Luchas sociales y conflictos interburgueses en la crisis de convertibilidad. Buenos Aires: Ediciones Continente.

Bonnet, A. (2011). Las relaciones entre estado y mercado: ¿un juego de suma cero? En A. Bonnet (comp.). El país invisible. Debates sobre la Argentina reciente. Buenos Aires: Peña Lillo / Continente.

Bonnet, A. y Piva, A. (2009). Introducción. En: Bonnet, A. y A. Piva. Argentina en pedazos. Luchas sociales y conflictos interburgueses en la crisis de convertibilidad. Buenos Aires: Ediciones Continente.

Bonnet, A., y Piva, A. (2013). Un análisis de los cambios en la forma de estado en la posconvertibilidad. En J. Grigera, (comp.). Argentina después de la convertibilidad (20022011). Buenos Aires: Imago Mundi.

Carrasco, C. (1991). El trabajo doméstico. Un análisis económico. Madrid: Ministerio de Trabajo y Seguridad Social.

Dalla Costa, M. (1977). "Las mujeres y la subversión de la comunidad". En James, S. y Dalla Costa, M. El poder de la mujer y la subversión de la comunidad, Siglo Veintiuno Editores.

Federici, S. (2013). Revolución en punto cero: trabajo doméstico, reproducción y luchas feministas. Madrid: Traficantes de sueños.

Fraser, N. (2015). Fortunas del feminismo. Quito: IAEN y Traficantes de sueños.

Gardiner, J. (2005) "El trabajo doméstico de las mujeres”. En Rodríguez, D. y J. Cooper, (comps.) El debate sobre el trabajo doméstico. Antología. México DF: UNAM.

Heller, L. (2000). El empleo femenino en los noventa ¿nuevos escenarios, nuevas ocupaciones? El caso argentino. Ponencia presentada en el XXII Congreso de Latin American Studies Association (LASA).

Hirsch, J. (2005). ¿Qué significa Estado? Reflexiones acerca de la teoría del Estado capitalista. Revista de Sociología e política (24), 165. 
Holloway, J. (1995). Un capital, muchos Estados. Revista Aportes para el Estado y la administración gubernamental (3).

MacKinnon, C. (1995 [1989]). Hacia una teoría feminista del Estado. Madrid: Ediciones Cátedra.

Marco Navarro, F. y Rodríguez Enríquez, C. (2010). Pasos hacia un marco conceptual sobre el cuidado. En: Montano Virreira, C. y C. Calderon Magana (coords.). El cuidado en acción: Entre el derecho y el trabajo. Serie Cuadernos de la CEPAL. $N^{\circ}$ 94. Santiago de Chile: Comisión Económica para América Latina y el Caribe (CEPAL).

Pateman, C. (1995 [1988]). El contrato sexual. México D.F.: Anthopos/UAM.

Piva, A. (2011). Una aproximación a los cambios en la Forma de Estado en Argentina (2002- 2009). Theomai: estudios sobre sociedad, naturaleza y desarrollo (23).

Rodríguez Enríquez, C. (2012) "La cuestión del cuidado: ¿el eslabón perdido del análisis económico?". Revista CEPAL, 106, abril de 2012.

Rodríguez Enríquez, C. (2015). Economía feminista y economía del cuidado. Aportes conceptuales para el estudio de la desigualdad. Nueva Sociedad (256).

Scott, J. (1996). El género: Una categoría útil para el análisis histórico. En: Lamas Marta (Comp.). El género: la construcción cultural de la diferencia sexual. México: UNAM-PUEG.

Seccombe, W. (2005). El trabajo de la ama de casa en el capitalismo. En Rodríguez, D. y J. Cooper (comps.). El debate sobre el trabajo doméstico. Antología. México DF: UNAM.

Valobra, A. (2015). El Estado y las mujeres, concepciones en clave feminista. Estudios Sociales del Estado, 1(2), 33-57.

Vega, C., y Gutiérrez Rodríguez, E. (2014). Nuevas aproximaciones a la organización social del cuidado. Debates latinoamericanos Presentación del Dossier. Íconos: Revista de Ciencias Sociales (50).

Zibecchi, C. (2014). ¿Cómo se cuida en Argentina?: definiciones y experiencias sobre el cuidado de niños y niñas. Ciudad Autónoma de Buenos Aires: Equipo Latinoamericano de Justicia y Género - ELA.

\section{Bases de datos:}

CEPALSTAT. Bases de datos y publicaciones estadísticas de la CEPAL.

INDEC (2013) Encuesta sobre Trabajo No Remunerado y Uso del Tiempo. Disponible en: https://www.indec.gob.ar/nivel4_default.asp?id_tema_1=4\&id_tema_2=31\&id_tema_3=117 